\title{
'Symbiocracy': The Structuring of New Societies in Space Based on the Principles of Mutualism and Symbiotization
}

\author{
Elizabeth Song Lockard ${ }^{1}$ \\ University of Hawaii, Honolulu, Hawaii, 96813
}

\begin{abstract}
In the absence of any pre-existing social infrastructures in Space-as well as inhabiting an environment from which we did not evolve-the crucial role of any proposed social institution will be above all the survival of the first Space settlers. Therefore all factors critical to survival must be exhaustively identified and considered, as any oversights could result in extinction of the settlement. The social institutions that currently exist on Earth will not be viable for Space societies, as there is no existing social infrastructure to build upon, and physical conditions are not like anything we have evolved from or have experienced before. Unlike on Earth, the model for life in Space must be developed as a seminal one. This initial pioneer community will be in effect the Noah's Ark to the solar system.

The purpose of this paper is to argue that prevailing social structures based on hierarchy, competition, and conquest must be cast aside in favour of models based exclusively on a cooperative and collaborative system of networks. No longer can we think in terms of conquering Space, but instead co-habitating with it. The notion of symbiocracy, derived from a combination of the biological processes of symbiosis and the socio-political principles of democracy, will be central to this model. It is a form of governance which acknowledges not the right to happiness, but the necessity of all living things to seek fulfillment; it doesn't promote survival but robustness. And unlike current models of democracy which are based on the assumption of equality, symbiocracy is founded instead on the premise of vitality. Venturing into Space will instead give humanity the extraordinary opportunity to design new ways of living which could never have been feasible on Earth.
\end{abstract}

\section{Introduction}

$\mathrm{T}$ He criteria for Space settlements are qualitatively very different from those of long-term missions. Whereas missions have specific scientific objectives, settlements will be more concerned with liveability in Space, and thus will be based on criteria for optimization rather than survival tolerances. As such they cannot be considered as quantitative extensions of long duration missions. Optimization criteria should address not only the physical conditions of the habitat but the social conditions as well.

Because delayed communications between the crew and mission control will be a part of life in more remote locations such as Mars, command from an Earth base will no longer be viable for overseeing and regulating the activities of the colony. A Space colony, especially a distant one, will have to function more autonomously, but this isolation will also necessitate adaptation to the new environment.

New models to regulate social interaction should be considered; given the degree of independence of a remote Space colony, what kind of self-governing strategies will be appropriate and effective? This paper will discuss an alternative to the authoritarian-based structures, reminiscent of rigid militaristic regimes, that could better regulate interaction between individual Space settlers, their counterparts on Earth, as well as between multiple settlements in the future.

Developing new paradigms for social interaction in Space is not only crucial for the more immediate practical applications to a seminal colony, but also to the relationships between subsequent multiple colonies, as well as with other life forms we may encounter beyond the boundaries of Earth. Though still speculative at this time, a protocol for first encounters with other life forms - whether deemed intelligent or not - should be established prior to such an event.

${ }^{1}$ Ph.D. Candidate, Department of Political Science, Hawaii Research Center for Futures Studies, Saunders Hall, AIAA Student Member. 
The need for governance can be assessed by the anticipated degree of social conflict to be mitigated. A state of social harmony does not call out for the intervention of governance--only those situations which require resolution. Isolated and confined environments, as well as remote and extreme ones provide excessive social and physical stressors which must be thoroughly addressed if the colony is to survive. Therefore, it would be imprudent to limit ourselves only to the most virtuous stratum of human behavior and nature; we must consider the wide spectrum of actions, both benevolent and deviant, however those may be defined. The philosophies of Hobbes and of Plato speak to this dichotomy: the human race is both violent and brutish in its most base condition, as well as noble and good at its most ascendant. Thus humanity should not only strive for the ideals which can be imagined, but also address our primal shortcomings as well. The question I have therefore posed is: how can a socio-political structure elicit humanity's greatest potential, at the same time minimizing, or even helping us transcend our deficiencies?

Since a strategy for realization is only as strong as its conceptual foundation, I chose to focus first on developing a new cosmological basis for a political design--using analogs and theories from the fields of biology, evolution, and physics--upon which then strategies for implementation of governance structures would be derived.

\section{Proposal Parameters}

Locale:

Settlements and long-term communities on Mars or other more remote locations

\section{Size/Scale:}

Unit size $=15-20$ individuals

(based on the biological model of extended family or clan)

Maximum collective size $=$ several hundred to a few thousand (based on the limits of perception; the largest number that still retains a sense of cognitive cohesion)

It is important that a governance system is neither too localized nor too general but instead addresses both a plurality as well as an extreme range of scales. The reasons for this are: 1) the maximization of diversity in both scales and groups; 2) the development of relationships, connections, and interdependence at the different levels; 3) the creation of multi-perspectual and transdisciplinary realms based on systems theory, which go beyond reductivist solutions by encouraging more complex dynamic processes. In addition, by addressing various scales, a system of governance can respond to the need for both intimacy and anonymity, empathy and alienation, public and private life.

\section{Timeframe:}

To be implemented at the same time as the first colonies are formed.

\section{Scope of applicability:}

The governance system is intended to be valid until the principles of symbiosis and quantum cosmologies are supplanted by more progressive and more complex ontological paradigms that reflect evolved realities.

\section{Cosmological Foundations}

Life and its environment grow increasingly complex synchronously. According to the Gaia hypothesis, life doesn't simply react to its environment, but alters it as well, so that both life and its environment are constantly responding and adapting to each other. As life evolves it also participates in the evolution of its environment, just as a changing environment participates in the evolution of life. In the same way that the notion of the entire Earth as a large, self-sustaining feedback or ecosystem was an extrapolation of regionalized biosystems, Gaian principles could similarly be extrapolated to the realm of the solar system ${ }^{2}$; in the future science may uncover the same relationships on even larger (cosmic) scales.

${ }^{2}$ Within the solar system, planetary conditions are a result in large part of their relation to other planets, as well as, of course, their proximity to the Sun. One obvious example of this is the gravitational forces exerted by each planetary mass. In the case of the Earth, the gravitational force of the Moon is responsible for our planet's tidal changes, just as Earth's gravity keeps the Moon in our orbit. The immense heat within the moon Io - too far away to be warmed by the Sun - is due to frictional stresses caused by its proximity to Jupiter. 
The Earth, according to Lovelock's theory, is not stable as we observe at any given moment, but rather in a state of constant disequilibrium. It is perhaps best characterized as a process of dynamic stabilization; rather than a state of instability. Lovelock concludes that disequilibrium is evidence for the existence of the processes of life and that equilibrium is conversely indicative of its absence. Life therefore can be characterized as a process of striving for stability.

From a Darwinian perspective, natural selection does not take into account the impact that life has on its environment, only how the changing environment impacts living things. Organisms are reactive and adaptive, whereas the Earth itself remains unyielding. Natural selection is essentially a process that promotes survival of only the fittest through competition and conquest. It results in privileging a few, over the majority, not only for the right to survival but also for the opportunity to reproduce in order to pass on its genes. In this model there is no justification for self-sacrificing acts of compassion or altruism, as that type of behavior is seen as detrimental to the interests of the individual.

The principle of competition viewed within an evolutionary context is an essential aspect of natural selection. In much of the animal world, members of a species (generally males) compete in order to win the 'privilege' to procreate; theoretically, those who are strongest or superior defeat those who are inferior and weak, thus increasing the chances of the survival of the species through the transmission of superior genes to the offspring. The end result within any given group however, is the ascendance of some based on the descent of the majority. Success and evolutionary fulfillment are therefore only for a select few. While this process may still hold true, it is however, no longer sufficient in explaining the behavior of life.

As Darwin himself states in "The Descent of Man", humans have developed 'social sympathies' - the antithesis of competitive behavior that is mandated by natural selection. We not only take care of our own progeny, but also of others that are not related to us genetically. We protect the weak and the infirm; we extend rights to animals. These traits are pronounced in more complex species. This behavior - what we call humane - ostensibly contradicts the notion of natural selection.

It stands to reason then that not only do living entities evolve, but the processes of evolution themselves as well. If the tenets of evolutionary theory describe the development of living organisms, then it should follow that they are applicable to the development of the processes of life as well. As life evolves towards greater complexity and adaptability, then so must its concomitant principles and processes. Species are less prone to extinction if they diversify functionally. Not only then does the organism itself evolve, but its functions and its interface with the environment change along with it.

If evolution leads to greater diversity in living organisms as a means to insure survival, then the evolutionary processes themselves must also increasingly diversify to insure that greater complexity. If this is true then it is possible that competitive behavior among human beings is becoming an obsolete way to progress as a species. Rather than signifying the end of evolution however, it may instead reflect a new epoch in the processes of evolution (or 'meta-evolutionary' processes).

Competition as a function of the struggle for life however, is an inherently problematic notion. While competitive endeavor arguably brings out human virtues, spurring us on to great discoveries, accomplishments, and wondrous physical feats, competitive behaviors have also nurtured - a darker side of humanity, often resulting in conflict, domination, violence, and unethical behavior. Isn't our drive to compete, the underlying motive of which is to demonstrate superiority, also incongruous with the notion of equality that is cherished in western democratic cultures? If competition is both a source of conflict as well as a source of challenge, are there other ways that are less destructive to exploit our potential, or to create challenge that encourages growth?

Finally, must competition even be an essential part of human nature or fundamental to our evolutionary development? Homo sapiens haven't significantly evolved biologically in the last 40,000 years $^{3}$, so it is debatable to what degree biological evolution is still relevant to our future development. Significant evolution appears to occur more gradually in more complex organisms, as change in environment may not warrant adaptation to the same degree that it may necessitate it in organisms of lesser complexity. Instead, cultural and technological evolution have taken over where biology left off, implying that human constructs have the capability to influence, if not supersede, the forces of nature. Humans can consciously and willfully direct the course of their own evolution.

The increasing tendency towards what could be deemed as 'compassionate behavior' is a manifestation of an altruistic disposition not consistent with Darwin's natural selection, but can be explained in the context of symbiotic processes. In symbiosis, a single organism or an entire species behaves benevolently towards other members of, or

${ }^{3}$ Shostak, S.and Bennett, J., Life in the Universe 
outside, their species in order to serve their own interest. While altruism is not in keeping with the gene's disposition towards selfishness, reciprocal altruism - the very basis of symbiosis - serves the gene better than competition does. This aspect of mutualism is critical to the symbiotic function, which differs from competition in that benefit is not gained at the expense of the other. Natural selection "weeds out" organisms that are not competitively successful, both in terms of individual survival and perpetuation of a gene line; whereas symbiosis strengthens or reinforces collaboration between individual organisms (insuring immediate survival) and species (insuring long-term, collective survival), allowing for a greater number of relationships to develop than competitive practices do. Competition is nihilistic towards relationships, and by thinning out the population, its process is an inherently subtractive one. On the other hand, symbiosis is an essentially additive, relationship-building process.

'Compassionate' behavior is in fact a trait of symbiotic relationships, through which organisms or entire species benefit through mutual cooperation. It relies on diversification of organisms and species: the greater the diversity, the better chance not only to survive but to thrive and prosper (robustness). Not only is diversity a prerequisite for symbiosis to occur, but diversification of life can better withstand ecological crises or catastrophes. Moreover, a system made up of heterogeneous organisms can create far more complex relationships than homogeneous ones. Because of the dissimilarity of organisms that must cooperate with one another, the symbiotic process is a negotiative one. Homogeneity in organisms doesn't require this type of interaction; because each has nothing qualitatively different to offer the other, they either neutrally co-exist or they antagonistically compete for the same resources. The process of negotiation on the other hand, leads inevitably to reconciliation and adaptation, and is therefore the only process which can lead to growth. It need not be adversarial, but since the nature of growth is almost always traumatic, it most likely will be arduous and demanding.

According to biologist Lynn Margulis, "life did not take over the globe by combat, but by networking." She observes that greater evolutionary gains have been made though mutual cooperation than through struggle-in a sense 'out-competing' those who compete. In her view, symbiosis--not competition--is the major driver of evolution, because natural selection is neither sufficient to explain the apparent contradiction of cooperative behavior nor the genesis of entirely new species over the course of a single generation. Natural selection, which is based on individual competition in the interests of self-preservation and that of the individual gene ${ }^{4}$ (an independent and fragmented condition) can be characterized as a malevolent process, whereas symbiosis, which is based on mutual cooperation in the interests of collective preservation (an interdependent and relational condition) can be characterized as a benevolent one. Moreover, claims Margulis, "most evolutionary novelty arises from symbiosis" through a process of symbiogenesis ${ }^{5}$, implying that not only transformation, but transcendence, is a feature of ameliorative processes that simply cannot occur in ones based on conflict and adversity. With regards to eventual life in Space, she states: "Living together will be as crucial to the colonization of outer space as symbiosis and diversity were to the Paleozoic Era colonization of dry land. Life in space, if it is to occur, will require physical alliances, including new symbioses among differing life forms."

\section{beneficial}

mutualism: two different parties benefit and neither suffers

POSSIBLE OUTCOMES OF INTERACTION:

commensalism: one party benefits while the other suffers no disadvantage

break-even

neutralism: both parties are unaffected

parasitism: one party benefits while the other is harmed

\section{detrimental}

amensalism: disadvantageous to one party while the other is not affected

competition: both parties are harmed

$(--)$

Symbiosis can occur on a range of scales: it can occur between two different types of single-celled organisms such as bacteria which can actually exchange genetic information, and on the other extreme, it can occur between

\footnotetext{
${ }^{4}$ according to Dawkins' interpretation of Darwinism

${ }^{5}$ Margulis, L., Symbiotic Planet: A New Look at Evolution, p.33

${ }^{6}$ Ibid, p.106
} 
simple microbes and animals which are far more biologically complex. This transcendence of scale in symbiotic relationships is analogous to quantum phenomena as well. Manipulations or events that occur on a subatomic level appear to have effects that are not fully understood on much larger scales. Psi experience, entanglement, morphic resonance, and field theory are just a few examples of trans-scalar (as well as non-spatial) phenomena.

There are other parallels between symbiosis and quantum theory. In quantum cosmology all matter is interconnected, and so actions that affect one thing have universal repercussions. In symbiosis, the behavior of a single organism also has impact throughout an entire network of organisms. Both quantum theory and symbiosis are based on dynamic, relational, connected, holistic systems. If everything in the Universe is conceivably connected through quantum entanglement - and if the history of scientific discovery is any indication, there is a greater tendency towards connection over disjunction.

Evolutionary complexity, whether biological or technological, should then develop towards greater connective capacity; this in turn will lead to decreasing distinction or autonomy and increasing blurring of internal/external (or self/other) boundaries. This prescription is mandated by Margulis as well: "The near future of Homo sapiens as a species requires our reorientation toward the fusion and the merger of our planetmates that have preceded us in the microcosm." " Like Gaia, life and its environment are so interwoven that not only can one be understood only in relation to the other, but that the two are more indistinct than distinct. This idea is also reflected in conceptions of cosmic consciousness, quantum field theory, Vernadsky's concept of the noosphere, Radin's notion of an Integral Era, Margulis' theories of symbiosis, Kurzweil's Merged Epoch, Dator's notion of ubiquity, and the Japanese 'yaoyorozu'. Could this notion of ontological convergence possibly be extended to time and space as well, where past and future, or 'hereness' and 'thereness' are no longer distinct perceptions? Are we headed in the direction of omni-spatiality and omni-temporality?

This raises the question of whether laws of nature are subject to principles of evolution as well. Are they assumed to be a priori reality that we discover? Or do we - in the spirit of quantum cosmology - create more complex laws of nature to correspond to our own increasing complexity? Wasn't the classic Newtonian paradigm sufficient to describe the world at a time when it was in fact, far less complex? And then as life became more complex, the laws of nature had to develop accordingly — a kind of Gaia theory for physics. Or to re-phrase the words of Marshall McLuhan: The laws of nature shaped us, and thereafter we shaped them.

As demonstrated by quantum theory, the Universe is full of infinite potentialities which become actualized through consciousness, thus reality must be understood in the context of connections, relations, and process-a principle of reciprocating interaction between the physical and metaphysical realms that is analogous to the Gaia hypothesis. Vernadsky's and Teilhard de Chardin's notion of the universe as noosphere follows as an extension of Lovelock's theory of Earth as biosphere, but in the context of quantum physics where consciousness is proactive in creating reality. ${ }^{8}$ Life is evolving into higher states of consciousness, and the noosphere, as it evolves towards a more complex state of integration, represents the ultimate unification. Margulis writes: "Thinking of symbiosis is itself a symbiotic phenomenon", thus consciousness is already part of the trajectory towards greater symbiotic relationships.

\section{Governance Structures}

Symbiocracy is defined as a form of governance which acknowledges the necessity of all living things to thrive, based on a combination of the biological processes of symbiosis and the socio-political principles of democracy. Unlike current models of democracy however, which are based on the assumption of equality, symbiocracy is founded on the premise of vitality. Vitality in this context should not be confused with potency or any other connotative meanings associated with it, but understood simply as a condition of being vital or essential. Hence vitality distinguishes itself from equality in that it is a qualitative condition, one that implies value through indispensability; whereas equality - though often conceived in valuative terms - is fundamentally a quantitative

\footnotetext{
${ }^{7}$ Margulis, L., Symbiotic Planet: A New Look at Evolution, p.12

${ }^{8}$ However in the original conception, the formation of the noosphere referred only to human consciousness, which is limiting in its anthropocentrism and as such precludes the possibility of a cosmic consciousness that links all life collectively. Consciousness - or intelligence and compassion for that matter - are not exclusive traits of Homo sapiens, but rather of all life.

${ }^{9}$ Margulis, L., Symbiotic Planet: A New Look at Evolution, p.48
} 
condition that implies nothing more significant than neutrality between entities. The notions of indispensability, vitality, or relationality are not entailed by the definition of equality.

Moreover, a binding relationship or aspect of connectedness is not an inherent aspect of the notion of equality as it is in the notion of vitality, which entails mutual cooperation between entities for individual and collective benefit, and as such is an implicit condition of symbiotic relationships.

Democracy - which must acknowledge equality as an antecedent condition - is therefore limited to entities that are considered to be 'equal'. The measure of equality is in itself a problematic issue, even when the discussion is focused on human beings alone. Thus all entities that are not considered equal are excluded from the discourse. But because symbiocracy is not bound by the assumption of equality but rather by the aspect of vitality, it can be applied to all living things-animals, plants, even microbial organisms. It may upon first glance seem an absurd proposition to include things like bacteria in a governance system, but seen in the context of symbiotic relationships that are absolutely essential to maintaining the balance of life, it not only makes sense but actually becomes necessary.

To maintain its viability, the concept of democracy must also assume as a prerequisite a condition of rationalized altruism, a trait or behavior that is at best only inconsistently or sporadically exhibited. The notion of altruism is problematic as well, as it runs counter to theories of natural selection which tend to preempt acts of compassion or selflessness. Although as stated earlier, evidence indicates that life is evolving to become more compassionate, the innate tendency towards selfishness and self-preservation still cannot be disregarded.

While an act of unilateral altruism benefits one without necessarily benefiting the others (or even perhaps at the expense of the others), reciprocal altruism benefits all participants involved, and is therefore not only compatible with the theories of natural selection, but also promotes more complex relationships and networks between living entities. The gains are in fact greater than if each entity were to act solely on its own behalf. Thus symbiocratic structures take into account the welfare of both the individual and the collective, unlike democratic structures that protect only the rights of each individual without addressing the need for protecting the collective. In this way, symbiocracy integrates aspects of both democratic and socialist systems by incorporating both the rights of the individual and the responsibilities to the community, but in a manner that is consistent with our biological imperatives, not opposed to them. We need not rely on the principle of human rationality, flawed at best, as a basis for right action--which has been assumed since the Enlightenment to be the driving force behind moral behavior--as practical experience has shown no compelling correlation exists between the two.

The principles of symbiocracy provide a very different framework for evaluating the case for or against terraforming on Mars. No longer should we be thinking in terms of 'conquering' Space, but instead co-habitating within it. If microbial life exists on the red planet or elsewhere in the solar system, a symbiotic perspective will attempt to create mutually beneficial relationships with it, to find ways in which to integrate it into a larger ecosystem, rather than to extinguish it in deference to human needs. Discussions on the value of various life forms framed in terms of equality are no longer relevant in the context of symbiotization. Rather, all life forms will be acknowledged in terms of their vitality. While microbes may not be equal to us in terms of their individual complexity, they are superior to us in terms of their collective vitality and symbiotic capacity —in other words, we need them, but they don't need us! Symbiotization also addresses the issue of how to promote responsibility as well as protection of the ostensibly weaker members of society, two concerns which are at odds with our genetic tendency towards selfishness.

Lessons from Biosphere 2 show us that maintaining a critical mass of biological diversity is necessary for the entire ecosystem to thrive as well as to overcome crises. Though four thousand species were selected for containment, and countless others inadvertently interred, this degree of biodiversity was still not sufficient to maintain a self-stabilizing disequilibrium. Symbiotic relationships and processes will be absolutely essential for the viability of life in Space, where no localized planetary ecosystems currently exist but must be introduced from outside. They will be equally as critical should we discover new life forms beyond Earth as well. Like many Space-generated technologies that have had widespread terrestrial applications, understanding and developing symbiotic systems - ecologically, technologically, sociologically - will likely provide new paradigms for how to live back here on Earth as well. Though the focus of this paper is to examine the natural principles of biological symbiosis and how they can apply to social institutions such as structures of governance, they could also be considered in cultural and technological evolutionary contexts as well.

With its outward/extrospective focus on external information, technologies have consequently inhibited our proprioceptive abilities - namely our sensing of our selves - through a mediation of disengagement. The notion of 'self' in this context can be broadly interpreted at various scales, whether that be of the individual, of collective humanity, or of all living things. Technology applications then, must be used to enhance an inward/introspective focus in order to develop these innate capacities. It should serve to re-orient us towards an increasingly inclusive and expansive conceptualization of 'self'. 
Our relationship to technology, still informed by the Industrial era and Newtonian thinking, is essentially a mechanistic one. We use technology as machines to dominate and conquer our environment - seeing nature as an adversary to be vanquished - and in so doing, we have been increasingly distancing ourselves from the physical world we inhabit. In Space, where the environment is inhospitable to life, the technology of our habitats in insuring our survival, also threatens to reinforce that disjunction from our surroundings, which in turn hinders long-term adaptation. "Physical contact is a non-negotiable requisite for many differing kinds of life" ${ }^{10}$, therefore while remote technologies may supplement the ways in which we can interact, they can never supplant them entirely without alienating us not only from our essential physicality, but from evolutionary processes as well.

In an era of symbiotization, our relationship to technologies will become increasingly organic, utilized to create a greater empathy with our surroundings through engagement. Remote sensing, prosthetic devices, communications, and robotics, will present a wider range of symbiotic possibilities, and with that another level of complexity of convergence. Technology will also give rise to a new type of symbiosis: 'artificial symbiosis', where an organism interfaces with an artifact for mutual benefit (assuming that artifacts can benefit from such a merger). While biological symbiosis requires physical contact, cultural or technological symbiosis however, can be achieved both proximally and remotely.

To the extent though, that we are still physical creatures that reside in localized space means that some aspect of governance or any other social structure will have to acknowledge geography in terms of real space and real time. The experience of abstract spatiality through technology can supplement our physical experience of space but cannot replace it completely without alienating us from both our own physicality and the physical world surrounding us. Bioregion-based systems, such as the ahupuaha of Hawaii, through their immediacy and direct contact, lend themselves to fostering symbiotic interaction with the physical environment.

\section{Strategies for Implementation}

Four strategies are briefly outlined here as prerequisites for a symbiocratic system of governance.

\section{ABOLITION OF COMPETITION}

Competition is arguably only necessary for survival when there are limited resources. However, industrialized capitalistic societies still rely on economic competition, even when their needs have already been adequately met. Competition for the sake of itself is self-perpetuating, leading to excess consumption and hoarding of resources which in turn deprive others of satisfying their needs. It fosters societies of insatiable wants and an ethos of envy and dissatisfaction, which then leads to conflict. Because it is a zero-sum strategy, it creates a class of 'losers' where only one side can win at the expense of the other, and even those who 'win' are often themselves harmed in the process. With its sole focus on winning, the process of learning or of achievement is devalued.

Distinctions of superiority and inferiority bred by competitive behavior are not compatible with either the notions of equality or vitality that are coveted values in democratic systems. Achieving a democratic state is in fact, theoretically impossible where there exists hierarchical social structures.

To achieve symbiocracy, all competitive institutions will need to be eliminated or at least minimized so that new non-zero-sum strategies can be developed to replace them. There will therefore no longer be a class of losers, nor will there be a class of winners; the concepts of winning and losing simply will not exist. Motivations to excel will come from a different source: joy and passion for excellence and growth, and not from a sense of superiority and power over others. If we can attain this level of consciousness, the competitive traits we valued in the past will seem barbaric in retrospect. But this will be difficult, as the power and superiority won through competition is seductive.

\section{STRUCTURES OF COOPERATION \& COLLABORATION}

Competitive structures (hierarchical) will be replaced with structures that support and promote cooperative and collaborative behaviors (lateral). Processes will be based on networking and consensus instead of unilateralism or stratification of powers. In recognition of the importance of heterogeneity to make such networking systems mutually successful, diverse skills and talents will be cultivated and sought after, which implicitly acknowledges the contributions and value of all individuals. Preserving and promoting diversity rather than eliminating it will become the optimal way to socially evolve. The negotiative process entailed by the interactions between heterogeneous parties also fosters better communication and improves means of conflict resolution.

\footnotetext{
${ }^{10}$ Margulis, L., Symbiotic Planet: A New Look at Evolution, p.5
} 
The absence of independent endeavors may on the surface appear to compromise individual freedom. However, in a non-competitive world, the notion of freedom will no longer be synonymous with total independence. Its meaning will change significantly: freedom will not be viewed as diminished through interdependence but rather as enhanced through elimination of hierarchical control. The absence of imposed control will preempt conflict as well. Freedom cannot be enjoyed when essential needs are not first met, as is often the case in competitive scenarios. The satisfaction of both individual and collective needs will have an emancipatory effect, where the battle for survival is supplanted by the effort to achieve our higher values.

Continued Space exploration lends itself to promoting cooperative ventures because the technical know-how and the economic means for more ambitious projects cannot be borne by a single player, thus eliminating defection as an option. The future of Space exploration casts a long enough shadow that participants act from the perception that collaborative investment will yield gains for all the individual players for some time to come. If the shadow of the future is not sufficiently long however, then the incentive for cooperative behavior disappears.

In communities in Space, each individual should be responsible for some function of the colony that is indispensable to the survival of all; as the settlement expands, each subcolony will also retain an indispensable function relative to the entire collective community. Similar to the model at Los Horcones in Mexico, roles should rotate regularly so that all members develop the same expertise and a diversification of skills in order to be best equipped to handle catastrophe and crisis, as well as to keep stimulated and challenged.

Acts of charity which promote the welfare of others whose needs may not be met, are rewarded through social and economic incentives, such as additional leisure time, first choice of work duties, or vouchers for other benefits. In the highly vulnerable circumstances of the first Space colonies where survival will be a delicate and tenuous condition, rescue and charity missions will likely be prevalent.

\section{RECIPROCAL ALTRUISM/ MUTUALISM}

'Tit-for-Tat' - a collectively stable strategy - is considered to be the simplest as well as the most effective strategy to promote reciprocally altruistic behavior, both minimizing deviant behavior and rewarding compliant behavior. The strategy works best under circumstances where members of a community know each other (to maintain accountability) and where there is confidence that the timeframe for continued interactions is indeterminate, that is, always beyond the foreseeable future. Thus futures-oriented thinking is both a prerequisite as well as a consequence of this strategy.

The rules for the 'Tit-for-Tat' strategy are remarkably simple: the first move is to cooperate and then thereafter to repeat the previous move of the other player. It is a fundamentally friendly strategy--meaning that defection is never the default option. However, threat of retaliation or punishment must always be present in order to be effective and discourage repeated defection.

As well as being friendly, it is also a forgiving strategy in that if defection has occurred there is always a mechanism for quickly counteracting (rather than perpetuating) the defective behavior. Single defection is the means of retaliation; otherwise the default maneuver is cooperation. It is a strategy that promotes non-envy, which is crucial to maintain a stable symbiotic relationship, and since it is based on a non-zero sum proposition (meaning that there are no winners and losers), there is no such thing as competition or adversarialism. Research in games strategies concluded that the two primary traits of 'Tit-for-Tat' - niceness and forgiveness - proved far more successful in maximizing overall gains than games that were based on cunning or complexity.

Adversarialism requires two opposing parties, one representing 'us' and the other representing 'them'. This is precisely how the current American political system functions. All conflicts however, are only amplified if they are attempted to be resolved in this manner. Instead, conflicts should be resolved through a process of unification, one that endeavors to seek the most beneficial settlement for one party through the provision of benefits to the other. This favors a change-amplifying, non-zero sum process where the potentials are additive and limitless, over a change-counteracting, zero sum process where options are subtractive and finite.

\section{TECHNOLOGIES}

The role of technology since the advent of the machine has been to control and dominate our environment, which has led to increasing alienation from other life around us. In a system based on mutualism, there will be a shift from 'mechanizing technology' to 'organicizing technology', as technology will reflect the values and processes of symbiotic systems which emphasize fusion and merging instead of conquest and control. Examples of this will be remote sensing technologies, embedded technologies, and embodied virtuality, which will allow inhabitants in Space to sensually experience and engage their surroundings though they will not actually be able to physically occupy them. 
If sensory technologies will eventually merge us with our environments, inevitably blurring the distinction between subject and context, then it may also be conceivable that the mediation of technology in the human-human interface will blur the distinction between subject and subject. Surely then would divisive strategies of competition be completely antagonistic, as well as anachronistic.

Technology could also promote more responsible behavior and a greater capacity for foresight by creating interfaces that could assess range of risks and benefits from a particular action (individually and collectively, shortterm and long-term).

However, the danger here is that development of technology should not become an ends itself, as it has been in the previous machine age, but rather in the context of quantum theory and symbiosis so that it establishes greater connections--a 'mediation of integration'. The pursuit of technology for itself has also had a pre-emptive effect on establishing social goals. Rather than asking the question "what kind of social goals can be achieved through existing technologies?", we should ask "how should people live, and what technologies can then be developed to serve that purpose?"

\section{Conclusion}

Symbiocracy, a proposed concept for the government of new colonies in Space, is based on the principles of mutualism in which all participants benefit, and not only a select few. Unlike democracy, which emphasizes the right to the pursuit of happiness for the individual, symbiocracy acknowledges the necessity of all living things to seek fulfillment; like its biological counterpart upon which its principles are based, its goal is not to promote mere survival, but social robustness and cohesion.

While symbiocracy is a viable proposal for terrestrial governance, it presents an optimal model for life in Space, where an external authoritative regime is no longer practical. Since its most crucial role would be to insure the survival of the first contingent of Space settlers, there can be no distinction or hierarchy of benefits. As discussed in this paper, the best way to accomplish this is to create social relationships and networks based on cooperation and collaboration, instead of relationships based on competition--which inevitably results in deprivation for some or many participants.

Therefore, prevailing social structures here on Earth based on hierarchy, competition, and conquest must be cast aside in favour of models based exclusively on a cooperative and collaborative system of relationships. This also implies that we can no longer think in terms of conquering Space, but instead co-habitating with it, and with whatever life forms we may encounter beyond Earth. This transition will be facilitated by the act of relocation to Space itself, where humans will consequently see the Earth in a very different context. No longer constrained by terrestrial boundaries, their perceptual horizons will expand immensely. Earth will be seen anew, just as it did when

the first astronauts looked back at it from the moon. As a result of this profound displacement, humans will be able to adapt much more readily to radically new paradigms for living in the cosmos.

\section{References}

Margulis, L., Symbiotic Planet: A New Look at Evolution, Basic Books, New York, 1998.

Shostak, S.and Bennett, J., Life in the Universe, Pearson Addison Wesley, San Francsisco, 2007, Second Edition.

Margulis, L. and Fester, R., Symbiosis as a Source of Evolutionary Innovation, MIT Press, Cambridge, MA, 1991.

Dawkins, R., The Selfish Gene, Oxford University Press, Oxford, 1989.

Radin, D., Entangled Minds: Extrasensory Experiences in a Quantum Reality, Paraview Pocket Books, New York, 2006.

Rowe, A., "Will Biology Save the Universe?"

Lovelock, J., Gaia: A New Look at Life on Earth, Oxford University Press, Oxford, 1979.

Poynter, J., The Human Experiment: Biosphere 2, Thunder's Mouth Press, New York, 2006.

Kurzweil, R., The Singularity is Near, Penguin Group, New York, 2005.

Watson, D., "Theory of Enformed Systems"

Wheatley, M., Leadership and the New Science, Berrett-Koehler Publishers, San Francisco, 1994.

Slaton, C., "Quantum Theory and Political Theory"

Dator, J., "Towards Quantum Politics"

Aerts, D., "Quantum Democracy"

Dator, J., "Ubiquitous Society"

American Institute of Aeronautics and Astronautics

092407 
Zukav, G., The Dancing Wu Li Masters, Harper Collins Publishing, New York, 2001.

Chaisson, E., Cosmic Evolution, Harvard University Press, Cambridge, MA, 2001.

Gardner, J., Biocosm: The New Scientific Theory of Evolution, Inner Ocean Publishing, Maui, Hawaii, 2003.

Space Biospheres, the Orbit Foundation Series

Ward, P., The End of Evolution, Phoenix Giant, London, 1995.

Stone, C., "Should Trees Have Standing?"

Maruyama, M., Cultures Beyond Earth, Vintage Books, New York, 1975.

Maruyama, M., Human Futuristics, Social Science Research Institute, University of Hawaii, 1971.

Dudley-Flores, M. and Gushin, V., "On Our Best Behavior: Optimization of Group Functioning on the Early Mars Missions”

Axelrod, R., The Evolution of Cooperation, Basic Books, New York, 1984.

Morris, B., "Kropotkin's Ethical Naturalism"

Fehr,E., Fischbacher,U., Gachter,S., "Strong Reciprocity, Human Cooperation, and Enforcement of Social Norms"

Capra, F., The Web of Life, Anchor Books, New York, 1996.

Kropotkin, P., Mutual Aid: A Factor of Evolution, Dover Publications, Mineola, NY, 2006. 